\title{
COMMENTARY
}

\section{Feed the ICU patient 'gastric' first, and go post-pyloric only in case of failure}

\author{
Mette M Berger ${ }^{1 *}$ and Ludivine Soguel ${ }^{2}$ \\ See related research by White et al., http://ccforum.com/content/13/6/R187
}

\begin{abstract}
In a randomised trial comparing early enteral feeding by gastric and post-pyloric routes, White and colleagues have shown that gastric feeding is possible and efficient in the vast majority of critically ill patients. But the authors' conclusion that gastric is equivalent to post-pyloric is true in only the least severe patients. Given the extra workload and costs, post-pyloric is now clearly indicated in case of gastric feeding failure.
\end{abstract}

Randomised trials contribute to the determination of optimal nutritional treatment strategies. In a welldesigned study reported in the previous issue of Critical Care, White and colleagues [1] have investigated the impact of gastric versus post-pyloric (PP) route on early enteral feeding efficiency. Several interesting results are presented. First, the authors achieved a remarkable 80\% successful blind PP tube placement. They showed that the usual delay in initiation of PP feeding due to tube placement techniques [2] can be minimized by bedside tube placement by trained nurses. But although gastric enteral nutrition (EN) can be initiated faster (median 2.3 hours earlier than the PP), achieving the energy target 3.6 hours earlier, the difference is minor. The authors should be congratulated on a very efficient feeding protocol: to be able to initiate EN within 3 to 13 hours of admission and to achieve the target 3 to 5 hours later is great. Complications did not differ significantly between groups (pneumonias: 5 in the gastric group versus 11 in the PP group).

The authors attempted to solve the controversy of 'gastric versus post-pyloric' feeding in critical illness, after

\footnotetext{
*Correspondence: mette.berger@chuv.ch

'Service of Adult Intensive Care and Burns Centre, University Hospital (CHUV),

Rue du Bugnon 46, 1011 Lausanne, Switzerland

Full list of author information is available at the end of the article
}

several contradictory studies and two non-conclusive meta-analyses, by randomly assigning the patients to either feeding method from the start. They (apparently) observed a lower daily energy deficit, with trends toward smaller gastric residual volumes in the gastric group. Unfortunately, despite a good design, minimization regarding variables impacting on their main outcome, namely gastroparesis, was absent and the results are not as straightforward as claimed: the problem of group severity unevenness complicates the interpretation as in several other studies [3]. The authors were unlucky to enrol patients with a more severe condition into the PP group: the difference between median APACHE II (Acute Physiology and Chronic Health Evaluation II) scores of 24.5 and 30 is clinically relevant. Furthermore, to have more diabetics in the PP group is a worry as diabetes is associated with significant gastroparesis, the severity of which has motivated research for efficient prokinetics [4]. In the intensive care unit (ICU) patients in the severest condition (that is, patients with severe cardiovascular compromise on high-dose vasopressors), our group showed that the PP feeding resulted in a more efficient feeding and an additional $500 \mathrm{kcal}$ per day delivered compared with the gastric route [5]. A few studies in patients with major burns, in whom enteral feeding is strongly recommended, confirm the importance of severity of illness, with a more efficient feeding by the PP route in the severest patients. The commonest reason for gastric feeding failure is a large residual [6]: $83 \%$ of the 'failed' patients shifted on PP feeding achieve adequate feeding. Our group showed that computerized monitoring of energy delivery improved feeding in this category of patients [7], prompting the early use of PP feeding in case of large gastric residuals.

The study by White and colleagues [1] is characterized by a very low gastric feeding failure rate, with only four patients (7\%) requiring PP or parenteral feeding: this confirms the lower severity in this group as indicated by the APACHE scores. While the issue of severity is correctly discussed, the authors do not address the problem of diabetic gastroparesis. The difference in APACHE scores 
prompted them to analyse patients adjusted for severity and to analyse by intent-to-treat due to the 14 patients who were not fed according to random assignment (10 failures in tube placement and 4 failures in gastric feeding). Not surprisingly, the nutritional efficiency differences in favour of the gastric route disappear.

Despite these problems, the authors conclude that 'early post-pyloric feeding offers no advantage over early gastric feeding': we agree that this is certainly true in the general ICU population, but not in patients with pyloric dysfunction (that is, in the severest patients). We want to highlight the importance of not oversimplifying the interpretation of the results - such an oversimplification would be misleading - but of keeping the severity details in mind. This study is a serious contribution to the better usage of the feeding routes. On the basis of this study and others $[2,8]$, the good news is that the simplest feeding method is always worth trying. Feeding should be started by the gastric route, and given the extra workload and costs involved in gaining PP access, this procedure should be reserved for patients with high gastric residuals who fail gastric feeding within 48 to 72 hours of its initiation. This is early enough if energy delivery is monitored to prevent the build-up of an important energy debt [7,9].

\section{Abbreviations}

APACHE = Acute Physiology and Chronic Health Evaluation; EN = enteral nutrition; ICU = intensive care unit; PP = post-pyloric.

\section{Author details}

'Service of Adult Intensive Care and Burns Centre, University Hospital (CHUV), Rue du Bugnon 46, 1011 Lausanne, Switzerland

2University of Applied Sciences Western Switzerland (HES-SO), School of Health Professions Geneva, Nutrition and Dietetics Department, Rue des

Caroubiers 25, 1227 Carouge, Geneva, Switzerland

\section{Competing interests}

The authors declare that they have no competing interests.

Published: 19 February 2010

\section{References}

1. White H, Sosnowski K, Tra K, Reeves A, Jones M: A randomised controlled comparison of early post-pyloric versus early gastric feeding to meet nutritional targets in ventilated intensive care patients. Crit Care 2009, 13:R187.

2. Davies AR, Froomes PR, French CJ, Bellomo R, Gutteridge GA, Nyulasi I, Walker $R$, Sewell RB: Randomized comparison of nasojejunal and nasogastric feeding in critically ill patients. Crit Care Med 2002, 30:586-590.

3. Treasure T, MacRae D: Minimisation: the platinum standard for trials? Randomisation doesn't guarantee similarity of groups; minimisation does. BMJ 1998, 317:362-363.

4. Drenth JP, Engels LG: Diabetic gastroparesis. A critical reappraisal of new treatment strategies. Drugs 1992,44:537-553.

5. Berger MM, Revelly JP, Cayeux MC, Chiolero RL: Enteral nutrition in critically ill patients with severe hemodynamic failure after cardiopulmonary bypass. Clin Nutr 2005, 24:124-132.

6. Sefton EJ, Boulton-Jones JR, Anderton D, Teahon K, Knights DT: Enteral feeding in patients with major burn injury: the use of nasojejunal feeding after the failure of nasogastric feeding. Burns 2002, 28:386-390.

7. Berger MM, Revelly JP, Wasserfallen JB, Schmid A, Bouvry S, Cayeux MC, Musset M, Maravic P, Chiolero RL: Impact of a computerized information system on quality of nutritional support in the ICU. Nutrition 2006, 22:221-229.

8. Desachy A, Clavel M, Vuagnat A, Normand S, Gissot V, Francois B: Initial efficacy and tolerability of early enteral nutrition with immediate or gradual introduction in intubated patients. Intensive Care Med 2008 34:1054-1059.

9. Villet S, Chioléro RL, Bollmann MD, Revelly JP, Cayeux MC, Delarue J, Berger MM: Negative impact of hypocaloric feeding and energy balance on clinical outcome in ICU patients. Clin Nutr 2005, 24:502-509.

doi:10.1186/cc8862

Cite this article as: Berger MM, Soguel L: Feed the ICU patient 'gastric' first, and go post-pyloric only in case of failure. Critical Care 2010, 14:123. 